Vol. 59 (1999) [173-174]

\title{
Planar functions and related topics in finite fields
}

\author{
ROBERT S. COULTER
}

In this thesis we consider the problem of classifying planar functions over a finite field. We develop a method for constructing planar functions from known planar functions. This method produces planar functions which describe isomorphic planes. This result provides a natural way to define equivalence of planar functions in an algebraic and geometric sense. A class of polynomials which plays a significant role in the study of planar functions is the Dembowski-Ostrom polynomials. We show that DO polynomials are precisely those polynomials whose difference polynomials are affine. Before the work done in this thesis, the DO polynomials provided the only examples of planar functions over a finite field. We determine necessary and sufficient conditions for two particular types of DO polynomials to be planar. We then introduce a new class of planar functions. These functions describe a new class of Lenz-Barlotti type II projective planes, including the first such planes of non-square order.

We provide a group theoretic argument proving the well known result that a planar function cannot exist over an even ordered group. The argument leads us to define a class of polynomials which we call semi-planar. We define a functionally dependent incidence structure and show that the structure is a semi bi-plane if the function used is semi-planar. The DO monomial $X^{2^{\alpha}+1}$ is semi-planar on $\mathbb{F}_{q}, q=2^{e}$, if and only if $(\alpha, e)=1$. We show that, when $e$ is odd, a semi-planar DO monomial describes an association scheme with two associate classes. Such an association scheme is equivalent to a strongly regular graph. In this case we obtain Menon designs.

Weil sums are exponential sums of the form $\sum_{x \in \mathbf{F}_{q}} \chi(f(x))$ where $\chi$ is any additive character of $\mathbb{F}_{q}$ and $f \in \mathbb{F}_{q}[X]$. We consider Weil sums with $f(X)=a X^{p^{\alpha}+1}+b X$ where $p$ is the characteristic of $\mathbb{F}_{q}$. By generalising results of Carlitz we obtain explicit evaluations of these Weil sums for all cases in odd characteristic. We obtain similar results in characteristic two, up to the determination of a sign. These evaluations

Received 7 th October, 1998.

Thesis submitted to The University of Queensland November 1997. Degree approved April 1998. Supervisors: Dr Rex Matthews and Associate Professor George Havas.

Copyright Clearance Centre, Inc. Serial-fee code: 0004-9729/99 \$A2.00+0.00. 
are then used to determine the number of solutions $(x, y) \in \mathbb{F}_{q} \times \mathbb{F}_{q}$ of the equation $a x^{p^{\alpha}+1}+b x=y^{p^{t}}-y$ where $t$ is any integer dividing $(\alpha, e)$.

Department of Computer Science and Electrical Engineering

The University of Queensland

Queensland 4072

Australia 